\title{
Effect of fluid viscosity on elastic wave attenuation in porous rocks
}

\author{
Boris Gurevich*
}

\begin{tabular}{|c|c|}
\hline $\begin{array}{l}\text { ABSTRACT } \\
\text { Attenuation and dispersion of elastic waves in fluid- } \\
\text { saturated rocks due to pore fluid viscosity is investigated } \\
\text { using an idealized exactly solvable example of a system } \\
\text { of alternating solid and viscous fluid layers. Waves in pe- } \\
\text { riodic layered systems at low frequencies can be studied } \\
\text { using an asymptotic analysis of Rytov's exact dispersion } \\
\text { equations. Since the wavelength of the shear wave in the } \\
\text { fluid (viscous skin depth) is much smaller than the wave- } \\
\text { length of the shear or compressional waves in the solid, } \\
\text { the presence of viscous fluid layers requires a considera- } \\
\text { tion of higher-order terms in the low-frequency asymp- } \\
\text { totic expansions. This expansion leads to asymptotic low- } \\
\text { frequency dispersion equations. For a shear wave with } \\
\text { the directions of propagation and of particle motion in } \\
\text { the bedding plane, the dispersion equation yields the } \\
\text { low-frequency attenuation (inverse quality factor) as a } \\
\text { sum of two terms which are both proportional to fre- } \\
\text { quency } \omega \text { but have different dependencies on viscosity } \eta \text { : } \\
\text { one term is proportional to } \omega / \eta \text {, the other to } \omega \eta \text {. The low- } \\
\text { frequency dispersion equation for compressional waves } \\
\text { allows for the propagation of two waves corresponding }\end{array}$ & $\begin{array}{l}\text { to Biot's fast and slow waves. Attenuation of the fast } \\
\text { wave has the same two-term structure as that of the shear } \\
\text { wave. The slow wave is a rapidly attenuating diffusion- } \\
\text { type wave, whose squared complex velocity again con- } \\
\text { sists of two terms which scale with } i \omega / \eta \text { and } i \omega \eta \text {. } \\
\text { For all three waves, the terms proportional to } \eta \text { are re- } \\
\text { sponsible for the viscoelastc phenomena (viscous shear } \\
\text { relaxation), whereas the terms proportional to } \eta^{-1} \text { ac- } \\
\text { count for the visco-inertial (poroelastic) mechanism of } \\
\text { Biot's type. Furthermore, the characteristic frequencies } \\
\text { of visco-elastic } \omega_{V} \text { and poroelastic } \omega_{B} \text { attenuation mech- } \\
\text { anisms obey the relation } \omega_{V} \omega_{B}=A \omega_{R}^{2} \text {, where } \omega_{R} \text { is the } \\
\text { resonant frequency of the layered system, and } A \text { is a di- } \\
\text { mensionless constant of order } 1 \text {. This result explains why } \\
\text { the visco-elastic and poroelastic mechanisms are usually } \\
\text { treated separately in the context of macroscopic theo- } \\
\text { ries that imply } \omega \ll \omega_{R} \text {. The poroelastic mechanism dom- } \\
\text { inates over the visco-elastic one when the frequency- } \\
\text { indepenent parameter } B=\omega_{B} / \omega_{V}=12 \eta^{2} / \mu_{s} \rho_{f} h_{f}^{2} \ll 1 \text {, } \\
\text { and vice versa, where } h_{f} \text { is the fluid layer thickness, } \rho_{f} \\
\text { the fluid density, and } \mu_{s} \text { represents the shear modulus of } \\
\text { the solid. }\end{array}$ \\
\hline
\end{tabular}

\section{INTRODUCTION}

It is generally believed that the phenomena associated with the viscosity of the pore fluid represent one of the main causes of the attenuation of elastic waves in reservoir rocks and other fluid-saturated porous materials. However, despite decades of theoretical as well experimental research in this area, there is still some confusion as to the effect of the fluid viscosity on the attenuation at low frequencies. Indeed, according to Biot's theory of poroelasticity, dimensionless attenuation (inverse quality factor) in the low-frequency limit is proportional to frequency and to the inverse of viscosity (Biot, 1956a). On the other hand, the attenuation due to the local flow (squirt) mechanism is proportional to the product of frequency and viscosity (Mavko and Nur, 1975; O'Connell and Budiansky, 1977).
Despite the fact that the two mechanisms have the same basic physical cause (viscosity), there is as yet no sound theory that provides a comprehensive model that simultaneously includes both mechanisms. In this paper, I investigate the effect of pore fluid viscosity on elastic wave propagation using an idealized exactly solvable example.

One example which has proved particularly useful in various studies of porous media is a medium consisting of periodically alternating fluid and solid layers. Although such a configuration is obviously unrealistic, it possesses a number of key features of real porous materials (Rytov, 1956; Brekhovskikh, 1981; Schoenberg, 1984; Bedford, 1986). However, most of the research has been focused on ideal and low-viscosity fluids and relatively high frequencies when the layered system exhibits behavior typical of fluid-saturated media as described by the

Manuscript received by the Editor June 15, 2000; revised manuscript received February 16, 2001.

${ }^{*}$ Formerly The Geophysical Institute of Israel, P.O. Box 182, Lod 71100, Israel; presently Curtin University, Department of Exploration Geophysics, GPO Box U1987, Perth, Western Australia 6845, Australia. E-mail: gurevich@geophy.curtin.edu.au.

(C) 2002 Society of Exploration Geophysicists. All rights reserved. 
high-frequency asymptotic of Biot's theory of poroelasticity (Schoenberg, 1984; Schoenberg and Sen, 1986; Molotkov and Khilo, 1990; Molotkov and Bakulin, 1998). In this paper, I focus on low frequencies and relatively high-viscosity fluids.

The properties of waves in periodic layered systems at low frequencies can be studied using a low-frequency asymptotic analysis of the known exact dispersion equations (Rytov, 1956; Brekhovskikh, 1981). For the asymptotic analysis to be valid, the wavelengths of all the waves involved must be much larger than the spatial period of the periodic system. Since the wavelength of the shear wave in the fluid (viscous skin depth) is much smaller than the wavelength of the shear wave in the solid or of the acoustic wave in the fluid, the presence of viscous fluid layers requires a consideration of higher terms in the asymptotic expansions. The procedure is exactly the same for shear waves with the directions of propagation and of particle motion in the bedding plane, and for compressional waves propagating parallel to layering.

\section{LOW-FREQUENCY DISPERSION EQUATIONS}

Consider the system of periodically alternating solid and fluid layers of period $d$ shown in Figure 1. The elastic solid is characterized by density $\rho_{s}$, bulk modulus $K_{s}$, and shear modulus $\mu_{s}$. The viscous fluid is characterized by density $\rho_{f}$, bulk modulus (inverse compressibility) $K_{f}$, and dynamic viscosity $\eta$. The solid and fluid layer thicknesses are $h_{s}$ and $h_{f}=d-h_{s}$, respectively.

The aim is to derive the dispersion equations for shear and compressional waves propagating parallel to the bedding plane.

\section{Shear wave}

Consider propagation of a shear wave in the direction $x$ parallel to layering with the displacement in the direction $y$ normal to $x$ but also parallel to the bedding ( $S H$-wave). For a given frequency $\omega$, the solution of the mechanical problem can be sought in the form of a plane-wave particle displacement:

$$
u_{y}=u_{y 0} e^{i(a x-\omega t)}
$$

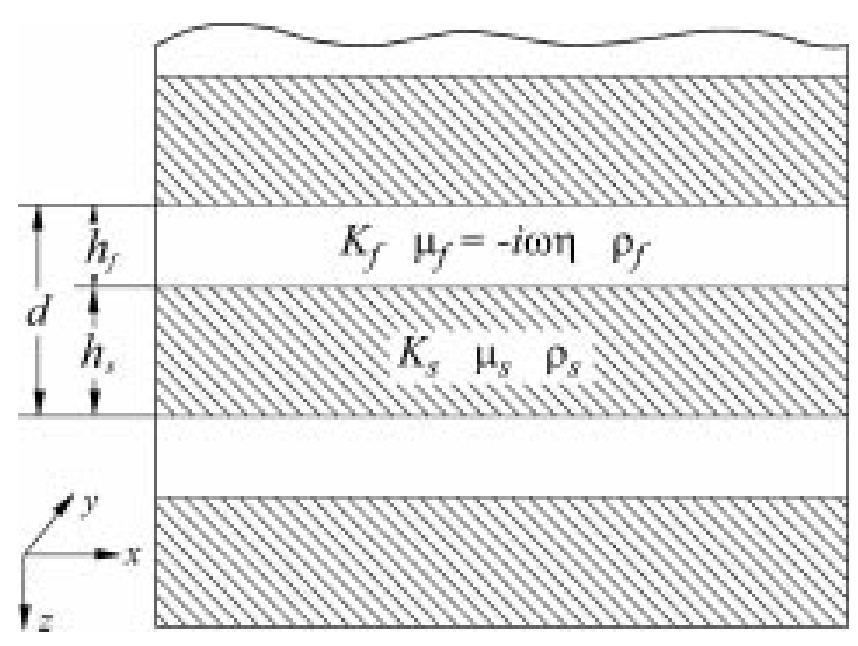

FIG. 1. Medium of alternating solid and viscous fluid layers.
We are looking for the low-frequency asymptotic of the wavenumber $a$ or the phase velocity $c=\omega / a$ as a function of $\omega$. To employ the known results for solid layered systems, one can regard the fluid as another solid with a complex shear modulus $\mu_{f}=-i \omega \eta$. $S H$-wave propagation in a periodic system of solid layers denoted $s$ and $f$ is governed by the exact dispersion equation (Rytov, 1956; Brekhovskikh, 1981),

$$
\begin{aligned}
& p\left[\left(\tan \frac{\beta_{s} h_{s}}{2}\right)^{2}+\left(\tan \frac{\beta_{f} h_{f}}{2}\right)^{2}\right] \\
& +\left(1+p^{2}\right) \tan \frac{\beta_{s} h_{s}}{2} \tan \frac{\beta_{f} h_{f}}{2}=0 .
\end{aligned}
$$

Here, $\beta_{s}^{2}=\omega^{2}\left(1 / c_{s}^{2}-1 / c^{2}\right)$ and $\beta_{f}^{2}=\omega^{2}\left(1 / c_{f}^{2}-1 / c^{2}\right)$, where $c_{s}=\left(\mu_{s} / \rho_{s}\right)^{1 / 2}$ and $c_{f}=\left(\mu_{f} / \rho_{f}\right)^{1 / 2}$ are shear velocities in the materials $s$ and $f$, respectively, and $p$ is given by

$$
p=\frac{\mu_{f} \beta_{f}}{\mu_{s} \beta_{s}} .
$$

For sufficiently long waves or low frequencies, the arguments of the tangents are small. Thus, the tangents in equation (1) can be replaced by their respective arguments. The resulting simplified equation can be solved analytically to give

$$
c^{2}=\frac{h_{s} \mu_{s}+h_{f} \mu_{f}}{h_{s} \rho_{s}+h_{f} \rho_{f}}
$$

or

$$
c^{2}=\frac{(1-\phi) \mu_{s}+\phi \mu_{f}}{\rho},
$$

where $\phi=h_{f} / d$ is the volume fraction of the fluid layers (porosity), and $\rho=(1-\phi) \rho_{s}+\phi \rho_{f}$ is the average density of the saturated rock. For the fluid layers the substitution

$$
\mu_{f}=-i \omega \eta
$$

yields the following expression for the velocity $c^{2}$

$$
c^{2}=\frac{(1-\phi) \mu_{s}}{\rho}\left(1-\frac{\phi}{1-\phi} \frac{i \omega \eta}{\mu_{s}}\right) .
$$

Due to the effect of viscosity, the velocity is now complex, implying the presence of attenuation.

Equation (4) is the result given in the literature (Brekhovskikh, 1981; Molotkov and Khilo, 1990) as a lowfrequency or long-wavelength approximation, with an obvious requirement that $\left|\beta_{s} h_{s}\right|$ and $\left|\beta_{f} h_{f}\right|$ must be small. However, at low frequencies, the wavelength of the viscous wave in the fluid is much smaller than that of the shear wave in the solid. Thus, the decrease of frequency $\omega$ also increases the relative magnitude of the terms containing $\beta_{f}$, so that higher-order terms in the power-series expansion of $\tan \left(\beta_{f} h_{f} / 2\right)$ may become significant. To analyze this phenomenon in greater detail, I retain the second term in this expansion, i.e.,

$$
\tan \frac{\beta_{f} h_{f}}{2} \simeq \frac{\beta_{f} h_{f}}{2}\left(1+\frac{1}{12} \frac{i \omega h_{f}^{2} \rho_{f}}{\eta}\right)
$$

Substituting this approximation for $\tan \left(\beta_{f} h_{f} / 2\right)$ while still replacing $\tan \left(\beta_{s} h_{s} / 2\right)$ by its argument and again solving for $c^{2}$ yields 


$$
c^{2}=c_{0}^{2}\left(1-\frac{i \omega \eta}{\mu_{s}} \frac{\phi}{1-\phi}-\frac{1}{12} \frac{i \omega}{\eta} \frac{\phi \rho_{f}^{2} h_{f}^{2}}{\rho}\right),
$$

where

$$
c_{0}=\lim _{\omega \rightarrow 0} c=\sqrt{\frac{(1-\phi) \mu_{s}}{\rho}} .
$$

The imaginary terms in the right-hand side of equation (6) indicate the presence of dissipation. The corresponding dimensionless attenuation (inverse quality factor) can be written

$$
q^{-1}=\frac{\operatorname{Im} c^{2}}{\operatorname{Re} c^{2}}=\frac{\omega \eta}{\mu_{s}} \frac{\phi}{1-\phi}+\frac{1}{12} \frac{\omega}{\eta} \frac{\phi \rho_{f}^{2} h_{f}^{2}}{\rho} .
$$

Equations (6) and (7) represent the asymptotic lowfrequency solution to the dispersion equation for the shear wave in a solid/viscous fluid layered system. The most interesting feature of these equations is the presence of two dissipative terms with the same frequency dependency but different dependencies on fluid viscosity. In fact, both terms are familiar ones. The first term (proportional to $\eta$ ) is the same as in equation (4) and accounts for the contribution of the complex shear modulus of the fluid to the overall complex shear modulus of the layered system (viscous shear relaxation). The second term, which scales with $\eta^{-1}$, can be identified with the visco-inertial attenuation in a porous medium as described by Biot's theory of poroelasticity (Biot, 1956a). In Biot's theory, the shear wave attenuation in the low-frequency limit is given by

$$
q_{B}^{-1}=\frac{\omega}{\eta} \frac{\rho_{f}^{2} \kappa}{\rho},
$$

where $\kappa$ denotes permeability. The permeability of a system of plain slits is (Biot, 1956b; Bedford, 1986)

$$
\kappa=\frac{\phi h_{f}^{2}}{12} .
$$

Substitution of equation (9) into equation (8) yields an expression identical to the second term in the right-hand side of equation (7).

One can see that both terms in equation (7) are related to the well-known mechanisms of wave attenuation in porous media: viscoelastic mechanism (viscous shear relaxation) (Mavko and Nur, 1975; O'Connell and Budiansky, 1977) and visco-inertial (poroelastic) Biot's mechanism (Biot, 1956a, b). In the above, both terms have been derived for an idealized porous medium from the same standpoint.

Figure 2 shows the result expressed by equation (7) against the numerical solution of the exact dispersion equation (1). The parameters of the medium were chosen such that the attenuation factors caused by the two mechanisms are of the same order of magnitude. This figure demonstrates that the combined effect of the two mechanisms as expressed by equation (7) represents the low-frequency asymptotic to the exact solution.

\section{Compressional waves}

The waves polarized in the $x-z$ plane can be analyzed in a similar fashion. The dispersion equation for waves symmetrical with respect to the middle axis of a layer is (Rytov, 1956; Brekhovskikh, 1981)

$$
\begin{aligned}
& 4\left(\mu_{s}-\mu_{f}\right)^{2} K_{1} K_{2}+\omega^{2} \rho_{s}\left[C^{2} \rho_{s}-4\left(\mu_{s}-\mu_{f}\right)\right] K_{2} \tan \frac{\beta_{s} h_{s}}{2} \\
& +\omega^{2} \rho_{f}\left[C^{2} \rho_{f}-4\left(\mu_{s}-\mu_{f}\right)\right] K_{1} \tan \frac{\beta_{f} h_{f}}{2} \\
& -\omega^{2} \rho_{f} \rho_{s} C^{2}\left[L_{1} \tan \frac{\beta_{f} h_{f}}{2}+L_{2} \tan \frac{\beta_{s} h_{s}}{2}\right]=0
\end{aligned}
$$

Here, $\alpha_{s}^{2}=\omega^{2}\left(1 / C_{s}^{2}-1 / C^{2}\right)$ and $\alpha_{f}^{2}=\omega^{2}\left(1 / C_{f}^{2}-1 / C^{2}\right)$, where $C_{s}=\left[\left(K_{s}+4 \mu_{s} / 3\right) / \rho_{s}\right]^{1 / 2}$ and $C_{f}=\left[\left(K_{f}+4 \mu_{f} / 3\right) / \rho_{f}\right]^{1 / 2}$ are compressional velocities in the materials $s$ and $f$, respectively, and

$$
\begin{aligned}
K_{1} & =\frac{\omega^{2}}{C^{2}} \tan \frac{\beta_{s} h_{s}}{2}+\alpha_{s} \beta_{s} \tan \frac{\alpha_{s} h_{s}}{2}, \\
K_{2} & =\frac{\omega^{2}}{C^{2}} \tan \frac{\beta_{f} h_{f}}{2}+\alpha_{f} \beta_{f} \tan \frac{\alpha_{f} h_{f}}{2}, \\
L_{1} & =\frac{\omega^{2}}{C^{2}} \tan \frac{\beta_{s} h_{s}}{2}-\alpha_{f} \beta_{s} \tan \frac{\alpha_{f} h_{f}}{2}, \\
L_{2} & =\frac{\omega^{2}}{C^{2}} \tan \frac{\beta_{f} h_{f}}{2}-\alpha_{s} \beta_{f} \tan \frac{\alpha_{s} h_{s}}{2} .
\end{aligned}
$$

The long-wave solution can be obtained by replacing all tangents with their respective arguments:

$$
C^{2}=\frac{1+4 \phi(1-\phi) \frac{\left(\mu_{s}-\mu_{f}\right)\left[K_{s}+\mu_{s} / 3-K_{f}-\mu_{f} / 3\right]}{\left(K_{s}+4 \mu_{s} / 3\right)\left(K_{f}+4 \mu_{f} / 3\right)}}{\rho\left(\frac{1-\phi}{K_{s}+4 \mu_{s} / 3}+\frac{\phi}{K_{f}+4 \mu_{f} / 3}\right)} .
$$

For viscous fluid layers with $\mu_{f}=-i \omega \eta$, this yields

$$
C^{2}=C_{0}^{2}\left(1-i Q_{V}\right)
$$

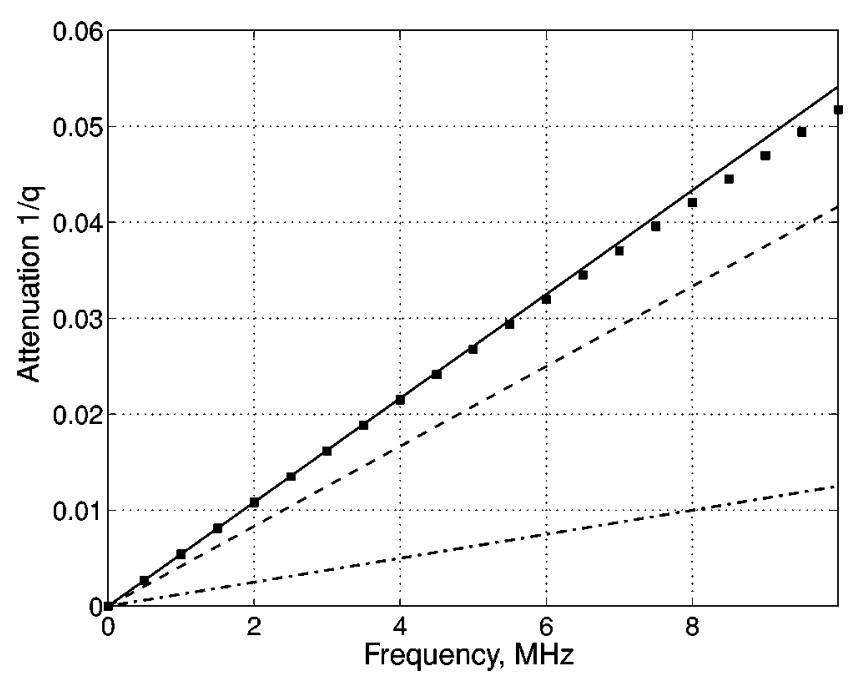

FIG. 2. Viscosity-related shear wave attenuation (inverse quality factor) as a function of frequency: numerical solution of equation (1) (squares) versus the low-frequency asymptotic. One can observe a perfect agreement between the numerical solution and the compound effect [equation (7), solid line] of the viscoelastic (dash-dotted line) and Biot's visco-inertial (dashed line) mechanisms. 
where

$$
C_{0}^{2}=\frac{1+4 \phi(1-\phi) \frac{\mu_{s}\left[K_{s}+\mu_{s} / 3-K_{f}\right]}{\left(K_{s}+4 \mu_{s} / 3\right) K_{f}}}{\rho\left(\frac{1-\phi}{K_{s}+4 \mu_{s} / 3}+\frac{\phi}{K_{f}}\right)}
$$

represents the velocity in the static limit, and

$$
\begin{aligned}
Q_{V}^{-1} & =\frac{4 \omega \eta \phi}{3 K_{f}}\left[\frac{K_{s}+4 \mu_{s} / 3}{(1-\phi) K_{f}+\phi\left(K_{s}+4 \mu_{s} / 3\right)}+(1-\phi)\right. \\
& \left.\times \frac{3 K_{f}\left(K_{f}-K_{s}+2 \mu_{s} / 3\right)-\mu_{s}\left(K_{s}+\mu_{s} / 3\right)}{4 \phi(1-\phi) \mu_{s}\left(K_{s}+\mu_{s} / 3-K_{f}\right)+\left(K_{s}+4 \mu_{s} / 3\right) K_{f}}\right]
\end{aligned}
$$

the attenuation. Equations (11)-(13) are valid in the highviscosity limit or, more precisely, when $\left|\beta_{f} h_{f}\right|$ is sufficiently small. For an arbitrary fluid viscosity, the low-frequency asymptotic analysis of equation (10) involving higher-order terms in the series expansion (5) yields an algebraic dispersion equation whose two roots represent two types of quasi-compressional waves. In the static limit $\omega \rightarrow 0$, one of these roots approaches a constant velocity $C_{0}$ ("fast wave"), whereas the other vanishes ("slow wave").

Fast wave.-The first root, which tends to $C_{0}$ in the static limit, is given by

$$
C_{1}^{2}=C_{0}^{2}\left(1-i Q^{-1}\right)
$$

where

$$
Q^{-1}=Q_{V}^{-1}+Q_{B}^{-1}
$$

with $Q_{V}$ given by equation (13) and

$$
Q_{B}^{-1}=\omega \frac{\rho_{f}^{2} \phi h_{f}^{2}}{12 \rho \eta}\left\{1-\frac{1}{\rho_{f} C_{0}^{2}}\left[\frac{1-\frac{2(1-\phi) \mu_{s}}{K_{s}+4 \mu_{s} / 3}}{\frac{\phi}{K_{f}}+\frac{(1-\phi)}{K_{s}+4 \mu_{s} / 3}}\right]\right\}^{2} .
$$

Equation (16) can be rewritten in the form

$$
Q_{B}^{-1}=\omega \frac{\kappa \rho_{f}^{2}}{\rho \eta}\left[1-\frac{\sigma M}{\rho_{f} C_{0}^{2}}\right]^{2}
$$

which is identical to the attenuation of the fast compressional wave in Biot's theory (Biot, 1962; Geertsma and Smit, 1961; Berryman, 1988). Here, I have used the expressions for Biot's constants charazterizing a system of alternating solid and fluid layers (Bedford, 1986):

$$
\begin{aligned}
\sigma & =1-\frac{2(1-\phi) \mu_{s}}{K_{s}+4 \mu_{s} / 3} \\
M & =\frac{1}{\phi / K_{f}+(1-\phi) /\left(K_{s}+4 \mu_{s} / 3\right)} .
\end{aligned}
$$

Slow wave.- The second root of equation (10) corresponds to a wave whose velocity vanishes in the zero-frequency limit:

$$
C_{2}^{2}=-i \omega\left[\frac{M}{\rho C_{0}^{2}} \frac{\mu_{s}\left(K_{s}+\mu_{s} / 3\right)(1-\phi) \phi h_{f}^{2}}{3 \eta\left(K_{s}+4 \mu_{s} / 3\right)}+\frac{\eta}{2 \rho_{f}}\right] .
$$

Equation (18) shows that the so-called slow wave is a diffusiontype dissipative wave. Similarly to the case of the attenuation of the shear and fast compressional waves, the most interesting feature of equation (18) is the presence of two terms with the same frequency dependency but different dependencies on fluid viscosity. The absolute value of $C_{2}^{2}$ as given by equation (18) is the sum of two terms: one which scales with inverse viscosity and one which scales with viscosity. In the limit of low viscosity, the second term vanishes, and the velocity is given by

$$
C_{2}^{2}=-\frac{i \omega \kappa(1-\phi) M}{\eta \rho C_{0}^{2}} \frac{4 \mu_{s}\left(K_{s}+\mu_{s} / 3\right)}{K_{s}+4 \mu_{s} / 3},
$$

which is identical to the expression for the complex velocity of Biot's slow wave (Biot, 1956a, 1962) for the medium of solid and fluid layers at low frequencies. For high viscosities, when the term proportional to $\eta^{-1}$ can be neglected, equation (18) has the form

$$
C_{2}^{2}=-\frac{i \omega \eta}{2 \rho_{f}}
$$

Equation (20) resembles the dispersion equation for the viscous wave in the free fluid.

\section{DISCUSSION}

\section{Characteristic frequencies}

The results obtained in the previous section give lowfrequency dispersion equations for shear and compressional waves in an idealized porous medium consisting of solid and fluid layers. For low fluid viscosity, these equations, as expected, reduce to the dispersion equations of anisotropic Biot's theory of poroelasticity. For high viscosity, these equations reduce to the classical equations of viscoelasticity. To further analyze these results, I rewrite one of the obtained equations [e.g., equation (7)] in the form

$$
q^{-1}=\frac{\omega}{\omega_{V}} \frac{\phi}{1-\phi}+\frac{\omega}{\omega_{B}} \frac{\phi \rho_{f}}{\rho}
$$

Here,

$$
\omega_{V}=\frac{\mu_{s}}{\eta}
$$

is the characteristic frequency of the viscoelastic mechanism. At this frequency the absolute value of the complex shear modulus of the viscous fluid equals the solid shear modulus. In turn,

$$
\omega_{B}=\frac{\eta \phi}{\kappa \rho_{f}}
$$

is Biot's characteristic frequency (Biot, 1956b), at which the wavelength of the shear wave (viscous skin depth in the fluid) equals the thickness of the fluid layers $h_{f}=\phi d$. The expressions for the two characteristic frequencies may be multiplied to give

$$
\omega_{V} \omega_{B}=A \omega_{R}^{2},
$$


where for shear waves

$$
A=\frac{3 \rho}{\pi^{2} \phi^{2}(1-\phi) \rho_{f}}
$$

is a dimensionless parameter of order 1 depending only on the porosity and the ratio of solid-to-fluid densities, and $\omega_{R}$ is the resonant frequency of the layered periodic system, at which the wavelength of the shear wave equals the period of the system,

$$
\omega_{R}=\frac{2 \pi c_{0}}{d} .
$$

Similarly to equation (21), the attenuation of the fast compressional wave, equation (15) can be written in the form

$$
Q^{-1}=S_{1} \frac{\omega}{\omega_{V}}+S_{2} \frac{\omega}{\omega_{B}},
$$

where $S_{1}$ and $S_{2}$ are dimensionless combinations of the material parameters of order 1 . Assuming that the bulk and shear moduli of the solid phase are of the same order of magnitude,

$$
K_{s} / \mu_{s}=O(1),
$$

we can see that the same is true for the compressional and shear velocities in the static limit

$$
C_{0} / c_{0}=O(1)
$$

Thus for the fast compressional wave, we can write an equation identical to equation (24)

$$
\omega_{V} \omega_{B}=A \omega_{R}^{2}
$$

where $A$ is given by an expression different from equation (25) while $\omega_{R}$ is still given by equation (26). Strictly speaking, for the fast compressional wave we should have used a different definition of $\omega_{R}$ based on $C_{0}$ instead of $c_{0}$, but the use of the same definition of the resonant frequency for both shear and compressional waves is possible in the order-of-magnitude analysis of the characteristic frequencies performed in this section. Also note that while our analysis of the compressional wave attenuation takes into account only the shear viscosity of the fluid, taking into consideration the bulk viscosity would be straightfoward and would have no effect on the order of magnitude of the characteristic frequencies, provided the bulk and shear viscosities are of the same order of magnitude, as is the case for most natural fluids (Landau and Lifshitz, 1987).

It is also instructive to note that frequency $\omega_{R}$, which is defined above as a resonant frequency for a periodic system with spatial period $d$, can also be thought of as the scattering frequency in an isotropic disordered (nonperiodic) porous material with the characteristic grain size $d$. Furthermore, while the relation between the characteristic frequencies [equation (24)] has been derived for a very specialized example of a periodic porous medium, one can surmise that similar relations (albeit with different numerical coefficients) would hold for a general porous medium with a single characteristic length or grain size. This gives some clue as to why the viscoelastic and visco-inertial mechanisms of attenuation are usually treated separately. Indeed, from equation (24), it follows that either

$$
\omega_{V}<\sqrt{A} \omega_{R}<\omega_{B}
$$

or

$$
\omega_{B}<\sqrt{A} \omega_{R}<\omega_{V} .
$$

In other words, if one of the characteristic frequencies is smaller than the resonant (scattering) frequency $\omega_{R}$, the other one is bound to be larger than $\omega_{R}$. But a macroscopic (effective medium) theory, by definition, aims to describe only the effects on spatial scales much larger than the grain or pore size (or period for periodic media), that is, for frequencies much smaller than $\omega_{R}$. Thus any macroscopic poroelastic theory can describe either the viscoelastic or the visco-inertial (Biot's) mechanism of attenuation. This has been shown for the idealized porous medium consisting of solid and fluid layers. For a general three-dimensional periodic porous medium with a single characteristic pore size, this fact was proved mathematically by Boutin and Auriault (1990) in the context of the theory of asymptotic homogenization of periodic structures, the theory that explicitly employs the ratio $\omega / \omega_{R}$ as a small parameter. In this paper, these terms have been obtained together only because the current approach is based not on any macroscopic (effective medium) theory, but on the dispersion equations (1) and (10) which are exact for all frequencies. And it was possible only because in our highly idealized example (periodic system of flat parallel layers) there is no scattering.

Furthermore, I define a fundamental parameter of the attenuation of shear and fast compressional waves in a layered solid/fluid system or a porous medium

$$
B=\frac{\omega_{B}}{\omega_{V}}=\frac{\eta^{2} \phi}{\mu_{s} \kappa \rho_{f}}
$$

that shows which of the two viscosity-related dissipation mechanisms dominates at frequencies $\omega \ll \omega_{R}$ when the macroscopic description makes sense (note that all the results in the previous section are obtained in the low-frequency limit, i.e., for $\omega$ which is much lower than the smallest of the three characteristic frequencies $\left.\omega_{B}, \omega_{R}, \omega_{V}\right)$. I emphasize that the parameter $B$ does not depend on the frequency, but only on the physical and geometrical properties of the layered system (or a porous rock). For the permeability of 1 darcy and viscosity of water, the parameter $B$ is about $10^{-4}$, but it may be larger for more viscous fluids (heavy oil, bitumen) and/or lower permeabilities. Let us consider three different situations:

1) $B \ll 1$. This situation is typical for high-permeability reservoir rocks and soils. In this case, Biot's poroelastic mechanism is dominant, and the viscoelastic terms in the dispersion equations are negligible.

2) $B \gg 1$. This situation is encountered for low-permeability materials (such as clays) and for porous rocks saturated with very viscous fluids (such as bitumen). In this case, seismic attenuation is controlled by the viscoelastic mechanism while the poroelastic effects are negligible

3) $B \sim 1$. This is an intermediate situation, when all three frequencies $\omega_{B}, \omega_{R}, \omega_{V}$ are of the same order of magnitude. Note that $\omega_{R}$ is primarily controlled by the dominant grain size of the rock, and thus is very high ( $>1 \mathrm{MHz}$ for grains smaller than $1 \mathrm{~mm}$ size). Given that the poroelastic and viscoelastic effects are controlled by the ratios $\omega / \omega_{B}$, $\omega / \omega_{V}$, it is clear that at low (seismic) frequencies both effects are negligible. However, these effects may become important for ultrasonic frequencies used in sonic logs and lab experiments. In this case, contributions of viscoelastic and poroelastic phenomena are comparable, 
and general relations (7), (15), and (18) which account for both phenomena should be used.

The three characteristic frequencies $\omega_{V}, \omega_{R}$, and $\omega_{B}$ introduce three dimensionless frequencies: $V=\omega / \omega_{V}=\omega \eta / \mu_{s}$, $\Omega=\omega / \omega_{R}=\omega d / c_{0}$, and $\ell=\omega / \omega_{B}=\omega \kappa \rho_{f} / \eta \phi$. Equation (24) shows that $V / \ell=A^{-1}(\Omega / \ell)^{2}=A(V / \Omega)^{2}=B$, and $\Omega^{2}=A V \ell$. The parameters $L=\sqrt{\ell}$ and $\Omega$ were introduced by Schoenberg and Sen (1986). Their work focused on the "low-frequency" $(\Omega \ll 1)$ but "small viscous skin depth" $(\ell \gg 1)$ regime, that is, on frequencies $\omega$ that are in the interval

$$
\omega_{B} \ll \omega \ll \omega_{R},
$$

which implies the medium with $B<1$. On the other hand, asymptotic low-frequency relations (6) and (7) obtained in this paper are valid when $V \ll 1$ and $\ell \ll 1$ at the same time, that is, when frequency is small compared with any of the characteristic frequencies

$$
\omega \ll \min \left(\omega_{B}, \omega_{V}\right) .
$$

As mentioned above, the viscoelastic and visco-inertial mechanisms of attenuation in porous media are usually treated separately. In particular, the viscoelastic phenomenon is ignored in Biot's theory by simply neglecting the fluid shear stress in the microscopic (pore-scale) constitutive equations. Pride et al. (1992) analyzed the effect of this approximation and showed that it requires that the parameter $V=\omega \eta / \mu_{s}$ be small. Indeed, if $V$ is very small, the viscoelastic attenuation is also very small [see equations (21) and (27)]. However, if at the same time the parameter $\ell$ is even smaller than $V$ (i.e., $\ell<V \ll 1)$, the poroelastic effects on the attenuation would be even less pronounced than the viscoelastic ones. The condition for neglecting the viscoelastic attenuation relative to the poroelastic one is $B=V / \ell \ll 1$. And, most importantly, this condition involves medium parameters only and is independent of the frequency. Thus, if this condition holds for a particular medium, Biot's theory would apply for all frequencies below the resonant frequency of individual pores. This is consistent with observations of Bedford (1986), who compared numerically the solutions of the exact dispersion equation for a layered solid/fluid system (with very small parameter $B$ ) with the prediction of Biot's theory, and found an excellent agreement in a wide frequency range. This is not surprising in the light of the results of Schoenberg and Sen (1986) and Molotkov and Bakulin (1998), who showed analytically that, in the case of low viscosity $B=V / \ell \ll 1$, the exact constitutive equations for a solid/fluid layered medium represent a partial case of anisotropic Biot's equations.

\section{Materials with multiple length scales}

All the results discussed above are valid for a medium with a single characteristic length parameter (layer thickness for idealized solid/fluid layered system, characteristic grain or pore size for a three-dimensional porous medium). However, it is known that many porous rocks have a wide spectrum of grain/pore sizes and heterogeneities of various scales. Moreover, it has been shown for some models of porous materials that the presence of more than one length parameter may have a significant impact on the elastic wave propagation. Two such models are of particular interest:

1) Porous medium with pores which have small aspect ratio $v \ll 1$. A pore with a low aspect ratio can be roughly characterized as having two characteristic sizes: length $d$ and thickness $v d \ll d$. A material containing such thin pores (or thin as well as rounded pores) is believed to exhibit so-called local-flow (squirt) attenuation mechanism associated with the wave-induced flow of the pore fluid within a thin pore (or from a compliant thin pore into a less compliant spherical pore) and vice versa. According to various analyses (Mavko and Nur, 1975; O'Connell and Budiansky, 1977; Jones, 1986), the characteristic frequency of the squirt mechanism can be written as

$$
\omega_{S Q}=\frac{G}{\eta} v^{3},
$$

where $G$ is the characteristic elastic modulus (of the solid or fluid). Clearly, $\omega_{S Q}$ as given by equation (30) resembles $\omega_{V}$ [equation (22)], but can be shifted substantially towards low frequencies. From this perspective, one can formally say that the squirt mechanism represents the classical viscoelastic mechanism shifted towards low frequencies by the presence of pores with very low aspect ratio. Clearly, this frequency shift invalidates the rigid relationship (24) between the characteristic frequencies. Understanding the role of the squirt mechanism requires a detailed and rigorous analysis of the relationship between various attenuation mechanisms and their characteristic frequencies in the media with low-aspect-ratio pores.

2) Macroscopically inhomogeneous porous medium, i.e., a micro-porous medium with some macroscopically heterogeneous structure. Examples of such media include a randomly layered porous medium (Gurevich and Lopatnikov, 1995; Gelinsky et al., 1998), a porous medium with macroscopic inclusions (Gurevich et al., 1998), and a double-porosity medium (Auriault and Boutin, 1994). Any such medium is characterized by two characteristic length parameters: a characteristic pore size $d$ and a characteristic size $b \gg d$ of the macroscopic heterogeneities (layers, inclusions, fractures). Macroscopic heterogeneity of these media causes an additional viscosity-related attenuation mechanism, associated with the flow of the pore fluid from more compliant to less compliant areas (or from the pores into fractures) and vice versa. This mechanism can also be described as the conversion of the fast wave energy into Biot's slow wave (by macroscopic heterogeneities), and a subsequent rapid dissipation of the latter. The characteristic frequency of this mechanism can be written as

$$
\omega_{M} \sim \frac{G}{\eta}\left(\frac{d}{b}\right)^{2}
$$

[note the similarity with equation (30)]. Depending on $b$, the characteristic frequency $\omega_{M}$ can be much lower than both $\omega_{B}$ and $\omega_{V}$, sometimes to such an extent that the seismic frequencies of $20-100 \mathrm{~Hz}$ can no longer be considered low frequencies with respect to $\omega_{M}$. Furthermore, such a medium is characterized by two scattering frequencies, 
which are associated with two characteristic length parameters $d$ and $b$, respectively. Clearly, a rigid relationship between the characteristic frequencies for macroscopically inhomogeneous porous media is no longer relevant. Analysis of the characteristic frequencies of the dominant attenuation mechanisms in heterogeneous porous media can be found in Gurevich et al. (1997), Gelinsky et al. (1998), and Shapiro and Mueller (1999).

\section{CONCLUSIONS}

Waves in periodic layered systems at low frequencies have been studied using an asymptotic analysis of Rytov's exact dispersion equations. This analysis leads to asymptotic lowfrequency dispersion equations. For a shear wave with the directions of propagation and of particle motion in the bedding plane, the dispersion equation yields the low-frequency attenuation (inverse quality factor) as a sum of two terms which are both proportional to frequency $\omega$ but have different dependencies on viscosity $\eta$ : one term is proportional to $\omega / \eta$, the other to $\omega \eta$. The low-frequency dispersion equation for compressional waves allows for the propagation of two waves which correspond to Biot's fast and slow waves. Attenuation of the fast wave has the same two-term structure as that of the shear wave. The slow wave is a rapidly attenuating diffusiontype wave, whose squared complex velocity again consists of two terms, which scale with $i \omega / \eta$, and $i \omega \eta$. For all three waves, the terms proportional to $\eta$ are responsible for the viscoelastic phenomena (viscous shear relaxation), whereas the terms proportional to $\eta^{-1}$ account for the visco-inertial (poroelastic) mechanism of Biot's type.

The characteristic frequencies of viscoelastic $\omega_{V}$, poroelastic $\omega_{B}$, and scattering $\omega_{R}$ attenuation mechanisms obey the relation $\omega_{V} \omega_{B}=\omega_{R}^{2}$, which explains why the viscoelastic and poroelastic mechanisms are usually treated separately in the context of macroscopic theories that imply $\omega \ll \omega_{R}$. The poroelastic mechanism dominates over the viscoelastic one when the frequency-independent parameter $B=\omega_{B} / \omega_{V}=$ $12 \eta^{2} / \mu \rho_{f} h_{f}^{2} \ll 1$, and vice versa. However, this balance can be changed by the presence in the medium of two or more scales of heterogeneity.

\section{ACKNOWLEDGMENTS}

I thank Jean-Louis Auriault, Michael Schoenberg, and Serge Shapiro for the in-depth discussions that inspired this work. The research was carried out with the support of the Israel Ministry of National Infrastructures.

\section{REFERENCES}

Auriault, J.-L., and Boutin, C., 1994, Deformable porous media with double porosity III: Acoustics: Transport in Porous Media, 14, 143162.

Bedford, A., 1986, Application of Biot's equations to a medium of alternating fluid and solid layers: J. Wave-Material Interaction, $\mathbf{1}$ 34-53.

Berryman, J. G., 1988, Seismic wave attenuation in fluid-saturated porous media: Pageoph, 128, 423-432.

Biot, M. A., 1956a, Theory of propagation of elastic waves in a fluidsaturated porous solid. I. Low-frequency range: J. Acoust. Soc. Am. 28, $168-178$.

1956b, Theory of propagation of elastic waves in a fluidsaturated porous solid. II. Higher frequency range: J. Acoust. Soc. Am., 28, 179-191.

1962, Mechanics of deformation and acoustic propagation in porous media: J. Appl. Phys., 33, 1482-1498.

Boutin, C., and Auriault, J.-L., 1990, Dynamic behaviour of porous media saturated by a viscoelastic fluid. Application to bituminous concretes: Internat. J. Eng. Sci, 28, 1157-1181.

Brekhovskikh, L. M., 1981, Waves in layered media: Academic Press.

Geertsma, J., and Smit, D. C., 1961, Some aspects of elastic wave propagation in fluid-saturated porous solids: Geophysics, 26, 169-181.

Gelinsky, S., Shapiro, S., Mueller, T., and Gurevich, B., 1998, Dynamic poroelasticity of thinly layered structures.: Internat. J. Solids Structures., 35, 4739-4752.

Gurevich, B., and Lopatnikov, S. L., 1995, Velocity and attenuation of elastic waves in finely layered porous rocks: Geophys. J. Internat. 121, 933-947.

Gurevich, B., Sadovnichaja, A., Lopatnikov, S. L., and Shapiro, S. A., 1998 , Scattering of a compressional wave in a poroelastic medium by an ellipsoidal inclusion.: Geophys. J. Internat., 133, 91-103.

Gurevich, B., Zyrianov, V. B., and Lopatnikov, S. L., 1997, Seismic attenuation in finely-layered porous rocks: Effects of fluid flow and scattering: Geophysics, 62, 319-324.

Jones, T., 1986, Pore fluids and frequency dependent wave propagation in rocks: Geophysics, 51, 1939-1953.

Landau, L., and Lifshitz, E., 1987, Fluid mechanics: Pergamon press, Inc.

Mavko, G., and Nur, A., 1975, Melt squirt in aesthenosphere: J. Geophys. Res., 80, 1444-1448.

Molotkov, L. A., and Bakulin, A. V., 1998, Effective model of solidfluid layers as a partial case of Biot model: J. Math. Sci., 91, 28122827.

Molotkov, L. A., and Khilo, A. E., 1990, On the equations of the effective model for an elastic medium with fractures filled with a viscous fluid: Voprosy Dynamicheskoj Teorii Rasprostraneniya Seismicheskikh Voln, 30, 88-95 (in Russian).

O'Connell, R. J., and Budiansky, B., 1977, Viscoelastic properties of fluid-saturated cracked solids: J. Geophys. Res., 82, 57195740 .

Pride, S. R., Gangi, A. F., and Morgan, F. D., 1992, Deriving the equations of motion for porous isotropic media: J. Acoust. Soc. Am., 92 3278-3290

Rytov, S. M., 1956, Acoustical properties of a thinly laminated medium: Sov. Phys. Acoust., 2, 68-80.

Schoenberg, M., 1984, Wave propagation in alternating fluid/solid layers: Wave Motion, 6, 303-320.

Schoenberg, M., and Sen, P. N., 1986, Wave propagation in alternating solid and viscous fluid layers: Size effects in attenuation and dispersion of fast and slow waves: Appl. Phys. Lett., 48, 1249-1251.

Shapiro, S. A., and Mueller, T., 1999, Seismic signatures of permeability in heterogeneous porous media: Geophysics, 64, 99-103. 